\section{Vivantes Hauptstadtpflege}

\section{Marketing-Offensive: Pflegekräfte gesucht}

"Die Vivantes Hauptstadtpflege wirbt ab sofort wieder großflächig um Mitarbeiter für die Altenpflege. In Berlin werden dazu mehr als 1.500 Werbeflächen Motive des kommunalen Pflegedienstleisters zeigen. Zudem wirbt die Vivantes Hauptstadtpflege auch

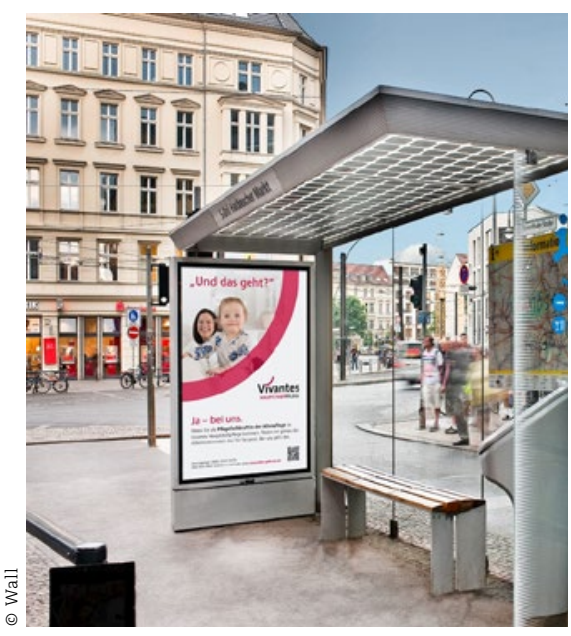

An Bushaltestellen, U-Bahnhöfen und Litfaßsäulen auf der Suche nach Mitarbeitern. auf Facebook und schaltet eine Karriereseite im Internet.

„Pflegefachkräfte und Pflegekräfte werden überall gesucht. Auch bei uns“, sagt Dr. René Herrmann, Geschäftsführer der Vivantes Hauptstadtpflege. „In insgesamt sechs Kampagnen-Motiven erläutern wir die Vorteile einer Mitarbeit bei der Vivantes Hauptstadtpflege. Ob flexible Arbeitszeiten, faire Entlohnung, Altersvorsorge oder Vergünstigungen, wir machen es möglich und sagen, , Ja - bei der Vivantes Hauptstadtpflege geht das“", meint Herrmann. Interessierte können sich dazu auch auf einer Kampagnen-Website unter www.hier-geht-das.de informieren und direkt bewerben. Dort finden sie auch ein Recruiting-Video, das einen authentischen Einblick in das Arbeiten bei der Vivantes Hauptstadtpflege gibt. Vivantes sucht sowohl Pflegefachkräfte als auch Pflegehelfer. Die Plakate sind an Bushaltestellen, UBahnhöfen und Litfaßsäulen zu sehen.

www.vivantes-hauptstadtpflege.de

\section{Hanse Institut Oldenburg}

\section{Neue Weiterbildungsstätte für Notfallpflege}

» Erstmals wird in Oldenburg eine Fachweiterbildung für die pflegerische Notfallversorgung angeboten. Die Deutsche Krankenausgesellschaft (DKG) genehmigte die Weiterbildungsstätte am Hanse Institut Oldenburg zum 1. Januar 2018. Ziel ist es, Pflegefachkräfte für die wachsenden Anforderungen in der Notfallversorgung zu qualifizieren. Weitergebildete Pflegekräfte sind Ansprechpartner für Patienten, nehmen Ersteinschätzungen vor und koordinieren die Versorgung. Berufserfahrene Pflegekräfte in Notaufnahmen können die Weiterbildung bis 2019 in verkürzter Form absolvieren. Der erste Kurs beginnt im Februar 2018. Das Institut kooperiert mit Krankenhäusern der Nordwest-Region. Träger sind das Evangelische Krankenhaus, das Klinikum und das Pius Hospital Oldenburg.

www.hanse-institut-ol.de

\section{Koblenzer Hochschulpreis}

\section{Masterarbeit zu gemeindenaher Pflege ausgezeichnet}

\) Die Wirtschafts- und Wissenschaftsallianz Koblenz vergab Ende 2017 erstmals den Koblenzer Hochschulpreis. Unter den Preisträgern war Sabine Erbschwendtner, Absolventin der Pflegewissenschaft an der Pflegewissenschaftlichen Fakultät der Philosophisch-Theologischen Hochschule Vallendar (PTHV). Sie wurde für ihre Masterarbeit „Soziale Praxis ambulant Pflegender im Kontext von Kommunikation und Dokumentation mit mobilen Endgeräten - Ergebnisse empirischer Erhebungen" geehrt. Wie steigende Bedarfe an guter Pflege in Deutschland zu bewältigen sind, sei bis heute ungeklärt, sagte Prof. Dr. Manfred Hülsken-Giesler, Prodekan der PTHV. „Eine [...] Strategie besteht darin, neue, computergestützte Technologien zur Unterstützung der Pflegearbeit bereitzustellen." Wie deren Einsatz die Pflegearbeit verändert, sei aber noch wenig bekannt. Die Arbeit liefere wichtige Einsichten.

\section{Arbeitgeber-Kampagne}

\section{UKE wirbt um neue Mitarbeiter}

" In einer groß angelegten Kampagne wirbt das Universitätsklinikum Hamburg-Eppendorf (UKE) um die besten Fachkräfte. Auf Plakaten, Postkarten und im Internet gewähren Mitarbeiter Einblicke in ihren Berufsalltag und zeigen, was das UKE als Arbeitgeber ausmacht. Mit der Aktion „Willkommen am Puls der Zeit." will das UKE vor allem Pflegekräfte, Ärzte und Wissenschaftler ansprechen.Eine Iris. Eine Fingerspitze. Haut. Die Detailaufnahmen geben zunächst Rätsel auf, erzählen dann aber besondere Geschichten. Es sind die Fingerspitze eines Pflegers, das Auge einer Ärztin und die Haut eines Forschers. Die UKE-Mitarbeiter zeigen, warum sie gern dort arbeiten. Die Motive sollen neugierig machen.

\section{www.uke.de/karriere}

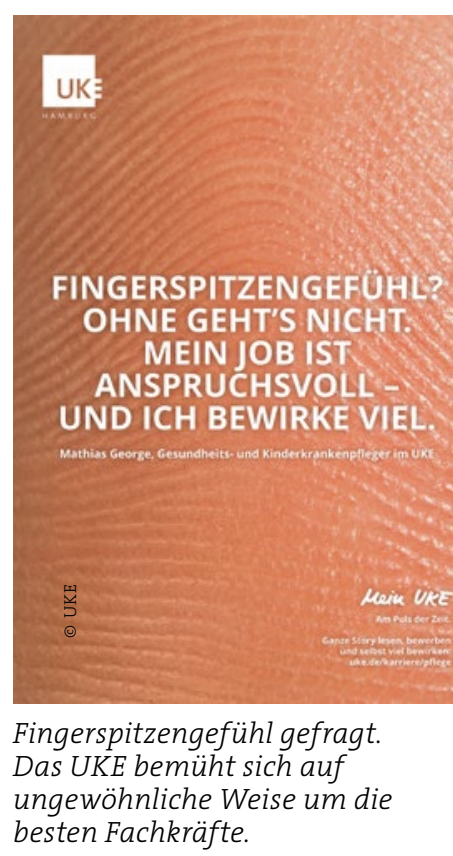

